\title{
Multipoint investigation of the source region of storm-time chorus
}

\author{
O. Santolík ${ }^{1,2}$, D. A. Gurnett ${ }^{1}$, and J. S. Pickett ${ }^{1}$ \\ ${ }^{1}$ Department of Physics and Astronomy, University of Iowa, Iowa City, IA 52242-1479, USA \\ ${ }^{2}$ Now at Faculty of Mathematics and Physics, Charles University, Prague, and at Institute of Atmospheric Physics, Czech \\ Academy of Sciences, Prague, Czech Republic
}

Received: 18 August 2003 - Revised: 2 February 2004 - Accepted: 25 February 2004 - Published: 14 July 2004

Part of Special Issue "Spatio-temporal analysis and multipoint measurements in space"

\begin{abstract}
In this case study we investigate the source region of whistler-mode chorus located close to the geomagnetic equator at a radial distance of 4.4 Earth radii. We use measurements from the four Cluster spacecraft at separations of less than a few hundreds of $\mathrm{km}$, recorded during the geomagnetic storm of 18 April 2002. The waveforms of the electric field fluctuations were obtained by the WBD instruments in the frequency range $50 \mathrm{~Hz}-9.5 \mathrm{kHz}$. Using these data, we calculate linear and rank correlation coefficients of the frequency averaged power-spectral density measured by the different spacecraft. Those coefficients have been recently shown to decrease with spacecraft separation distance perpendicular to the static magnetic field (Santolík and Gurnett, 2003) with a characteristic scale length of $100 \mathrm{~km}$. We find this characteristic scale varying between 60 and $200 \mathrm{~km}$ for different data intervals inside the source region. We examine possible explanations for the observed large scatter of the correlation coefficients, and we suggest a simultaneously acting effect of random positions of locations at which the individual chorus wave packets are generated. The statistical properties of the observations are approximately reproduced by a simple 2-D model of the source region, assuming a perpendicular half-width of $35 \mathrm{~km}$ (approximately one wavelength of the whistler-mode waves) for the distribution of power radiated from individual active areas.
\end{abstract}

Key words. Magnetospheric physics (waves in plasma, storms and substorms); Space plasma physics (waves and instabilities)

\section{Introduction}

Natural emissions of whistler-mode chorus consist of electromagnetic waves in the frequency range from a few hundred $\mathrm{Hz}$ to several $\mathrm{kHz}$. On time-frequency power spectrograms, they often appear as a sequence of intense, discrete wave packets. Each of them typically lasts for a fraction of a second and, during that time, its frequency changes

Correspondence to: O. Santolík

(ondrej.santolik@mff.cuni.cz) as a rising or falling tone. Chorus emissions have been known for several decades (see reviews by Omura et al., 1991; Sazhin and Hayakawa, 1992, and references therein) but their source mechanism is not yet well understood. It is generally believed that chorus is generated by a nonlinear process based on the electron cyclotron resonance of whistler-mode waves with energetic electrons, taking place close to the geomagnetic equatorial plane (e.g. Kennel and Petschek, 1966; Helliwell, 1967; Tsurutani and Smith, 1974; Nunn et al., 1997; Trakhtengerts, 1999). Storm-time chorus is especially important for the physics of the Earth's magnetosphere, since it can significantly influence the distribution functions of the energetic electrons in the outer radiation belt (e.g. Meredith et al., 2003; Horne et al., 2003; Horne and Thorne, 2003).

The chorus source region has been recently investigated using in situ measurements of the Polar and Cluster spacecraft, leading to new observations on various chorus characteristics, such as the position of the source (Lauben et al., 1998; LeDocq et al., 1998; Parrot et al., 2003), frequency shifts of chorus elements on power spectrograms obtained at different points in space (Gurnett et al., 2001), propagation directions of the chorus wave packets, and fine sub-packet structure embedded inside them (Santolík et al., 2003). The present paper is a sequel of the recent work on the correlation analysis of the power-spectral density of chorus measured in its source region (Santolík and Gurnett, 2003). The analysis was based on simultaneous measurement on board the four Cluster spacecraft during the geomagnetic storm of 18 April 2002. The correlation coefficient decreased in the plane perpendicular to the local static magnetic field $\left(\boldsymbol{B}_{\mathbf{0}}\right)$ with a characteristic scale of approximately $100 \mathrm{~km}$, while this scale was at least several times larger in the direction parallel to $\boldsymbol{B}_{\mathbf{0}}$. This was in agreement with the theory of Trakhtengerts (1999), who, in the direction perpendicular to $\boldsymbol{B}_{\mathbf{0}}$, predicted a characteristic scale between 100 and $300 \mathrm{~km}$ for spatial features of chorus generation. The theory also predicts that, in the direction parallel to $\boldsymbol{B}_{\mathbf{0}}$, the characteristic dimension of the source region is by an order of magnitude larger (Helliwell, 1967; Trakhtengerts, 1999). 
(a)
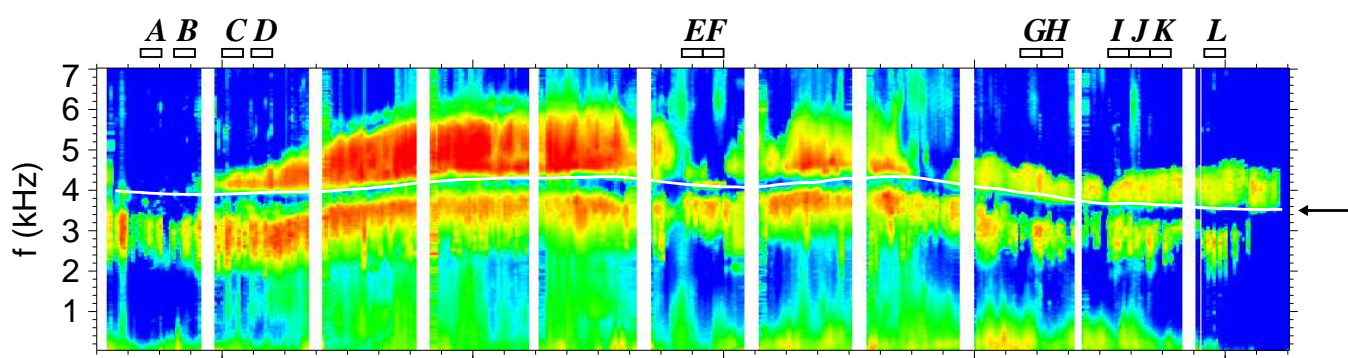

$1 / 2 f_{\mathrm{ce}}$

(b)
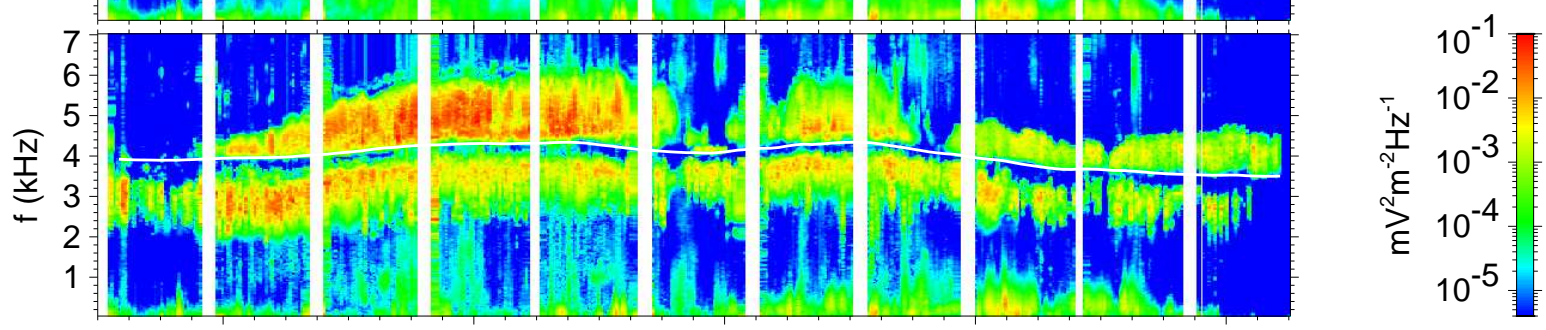

MLat (deg):

$\begin{aligned} R\left(R_{E}\right): & \\ \operatorname{MLT}(\mathrm{deg}) & \end{aligned}$

0846

0848

$-1.97$

4.38

0850

4.37

$-0.88$

0.21

21.01

0852

4.37

1.30

21.00
0854

4.37

2.39

20.98

Fig. 1. Data collected by the WBD instruments on 18 April 2002, in the source region of storm-time chorus. Average time-frequency power spectrogram of the electric field fluctuations measured by Cluster 1-4 is shown in panel (a). Mean absolute deviation of the power spectrograms from Cluster 1-4 is shown in panel (b). Universal time (UT) and position of Cluster 1 are given on the bottom using the radial distance $(R)$ in Earth radii $\left(R_{E}\right)$, magnetic dipole latitude (MLat) in degrees, and magnetic local time (MLT) in hours. One half of the local electron cyclotron frequency $1 / 2 f_{c e}$ is plotted over the panels $(\mathrm{a}-\mathrm{b})$. The letters A-L on the top indicate twelve 10 -second time intervals selected for the correlation analysis.

Santolík and Gurnett (2003) found relatively large variations of the obtained correlation coefficients when the data from several time intervals were combined. The aim of the present paper is to investigate these variations of the correlation coefficients, and to provide their tentative interpretation using a simplified 2-D model of the source region. Our investigation is based on multipoint measurements of the wideband (WBD) plasma wave instruments on board the four Cluster spacecraft (Gurnett et al., 1997, 2001). Supporting wave propagation parameters necessary to identify the source region are provided by the spectrum analyzers of the STAFF instruments (Cornilleau-Wehrlin et al., 2003), plasma density estimates are based on the data of the Whisper sounders (Décréau et al., 2001), and the electron cyclotron frequency is determined from measurements of the onboard fluxgate magnetometers FGM (Balogh et al., 2001). In Sect. 2 we show the overview of the WBD observations in the chorus source region and examples of high-resolution spectrograms. Correlation of the power-spectral density measured by the different spacecraft is then analyzed in Sect. 3. In Sect. 4 we interpret the results using a simple 2-D simulation of chorus source region, and, finally, in Sect. 5 we summarize the results.

\section{Time-frequency characteristics of chorus in the source region}

Wave measurements of the Cluster spacecraft during the the geomagnetic storm of 18 April 2002 (Dst index below
$-120 \mathrm{nT}$ and $K_{p}$ index of $7^{\circ}$ ) were recently studied in detail by Santolík et al. (2003). Here we only summarize their main features. Between 08:20 and 09:30 UT, the four spacecraft moved close to the equatorial plane on the nightside (MLT $\approx 21: 00$ ) at a radial distance of $4.4 R_{E}$. Their maximum separation was $\approx 260 \mathrm{~km}$ along $\boldsymbol{B}_{\mathbf{0}}$ and $100 \mathrm{~km}$ in the perpendicular plane. Intense chorus was observed by the WBD, STAFF, and Whisper wave instruments. Plasma density of a few particles per $\mathrm{cm}^{3}$, indicative of magnetospheric regions outside the plasmasphere, was estimated from the Whisper data (P. Canu, private communication, 2002; Canu et al., 2001). The WBD instruments measured continuous waveforms with pass-band filters between $50 \mathrm{~Hz}$ and $9.5 \mathrm{kHz}$, mainly using the $88-\mathrm{m}$ electric double-sphere antennas (for short periods of time, signals from magnetic search coil antennas were transmitted-these data are not shown here).

Figure 1 summarizes observations of the WBD instruments within the source region. Time-frequency power spectrograms of the electric field fluctuations measured by the four spacecraft are averaged and shown in Fig. 1a. The white line plotted over the spectrogram represents one half of the local electron cyclotron frequency $\left(1 / 2 f_{c e}\right)$ calculated from the average $\boldsymbol{B}_{\mathbf{0}}$ measured by the four spacecraft. Figure 1a shows two bands of chorus emissions, $\approx 2-3.5 \mathrm{kHz}$, and $\approx 4$ $6 \mathrm{kHz}$, separated by a gap of decreased power (Tsurutani and Smith, 1974) located below $1 / 2 f_{c e}$. The results are very similar on the four spacecraft but differences in details can be recognized. To give a quantitative measure of these differences we have calculated the mean value among the four 


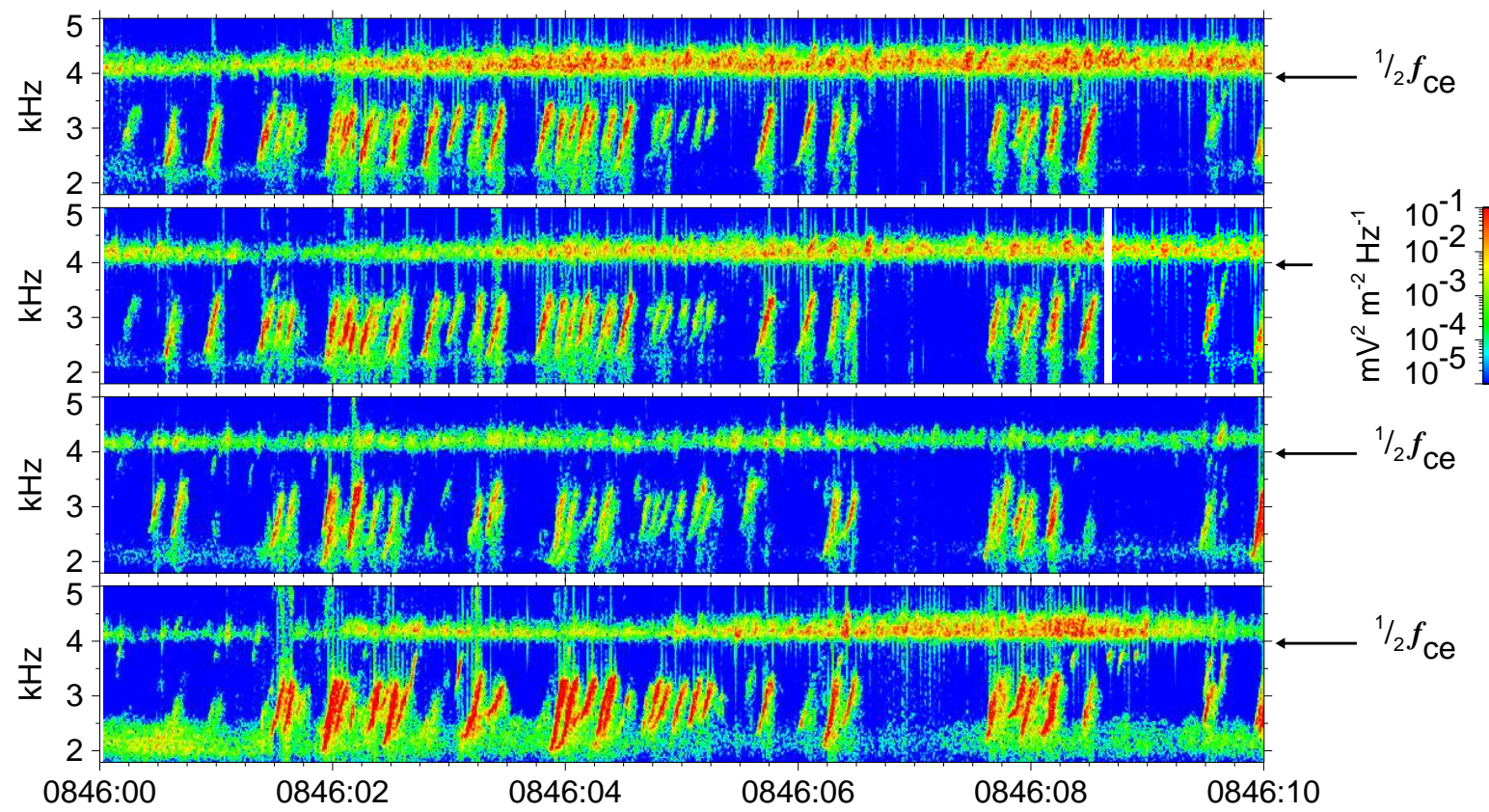

Fig. 2. Detailed time-frequency power spectrograms of electric field fluctuations recorded between 08:46:00 and 08:46:10 UT (interval C from Fig. 1). Panels (a-d) show the data from Cluster 1-4, respectively. Arrows indicate local $1 / 2 f_{c e}$ for each spacecraft.

absolute differences between each of the four spectrograms and their average. This mean absolute deviation is plotted in Fig. 1b. It shows that sometimes the differences can reach the same order of magnitude as the average spectrogram. This could reflect observations of the same but time shifted spectral features. We will show later on that this is not the case and that the high values of the mean absolute deviation directly reflect differences among the observed power spectrograms.

Simultaneous measurements of the STAFF-SA instruments show (see Fig. 1c of Santolík et al., 2003) that in the lower-frequency band of chorus (below $4 \mathrm{kHz}$ ), the Poynting flux predominantly has a southward component when the spacecraft are located to the south of a $4^{\circ}$-interval of magnetic latitudes around the equator. Similarly, the Poynting flux mainly has a northward component when measured to the north of this interval. The observed divergence of the Poynting flux has been interpreted as a passage through the chorus source region (Santolík et al., 2003). The source is located close to the equatorial plane, in agreement with previous results (LeDocq et al., 1998; Burton and Holzer, 1974; Burtis and Helliwell, 1969), and also with other Cluster observations (Parrot et al., 2003). The STAFF-SA data also show us that the average wave vectors were approximately parallel to $\boldsymbol{B}_{\mathbf{0}}$ in the source region (Fig. 1d of Santolík et al., 2003), as previously observed, e.g. by Hayakawa et al. (1984) and Goldstein and Tsurutani (1984). With the plasma density estimated from the Whisper data, and with the electron cyclotron frequency from the in situ FGM measurement, we obtain, using the cold plasma theory (e.g. Stix, 1992), a typical wavelength of approximately $30 \mathrm{~km}$ for a parallel propagating wave at $3.5 \mathrm{kHz}$.
From the data in Fig. 1, we have selected twelve 10-second intervals for further analysis (marked by letters A-L on the top of the figure). Examples are shown in Figs. 2 and 3. On these detailed power spectrograms, we can clearly see separate elements in the lower-frequency band of chorus below $1 / 2 f_{c e}$. They all appear as intense rising tones lasting for approximately $0.1 \mathrm{~s}$ and increasing their frequency from $\approx 2$ to $3.5 \mathrm{kHz}$. The upper band of chorus is at frequencies just above $1 / 2 f_{c e}$. It also contains rising elements which are sometimes (mainly in Fig. 3) synchronized with the elements appearing in the lower band. These individual elements in the upper band are better resolved in Fig. 3 but in both figures they are embedded in continuous hiss. The band below $1 / 2 f_{c e}$ also contains hiss at frequencies around $2 \mathrm{kHz}$, just below the lowest frequency of chorus. This is especially seen in Fig. 2d, where hiss seems to be directly connected to chorus (Koons, 1981; Hattori et al., 1991), triggering possibly the discrete elements (as discussed for another time interval by Santolík et al., 2003). These discrete elements are, nevertheless, always more intense than hiss by several orders of magnitude. If we now transform the measured electric field waveforms to acoustic waveforms using different loudspeakers for signals from the different spacecraft, the ensemble of these emissions sounds like a tropical forest, full of birds loudly chirping from different directions (lower band of chorus), and insects making their high-pitched sounds (higher band of chorus).

The chirps seem to come from different directions because, as can be seen in Figs. 2 and 3, the spectrograms from different spacecraft are similar but still slightly different. Moreover, this visual comparison shows more differences, for example, between Cluster 1 and 3 than between Cluster 1 
(a)

(b)

(c)

(d)

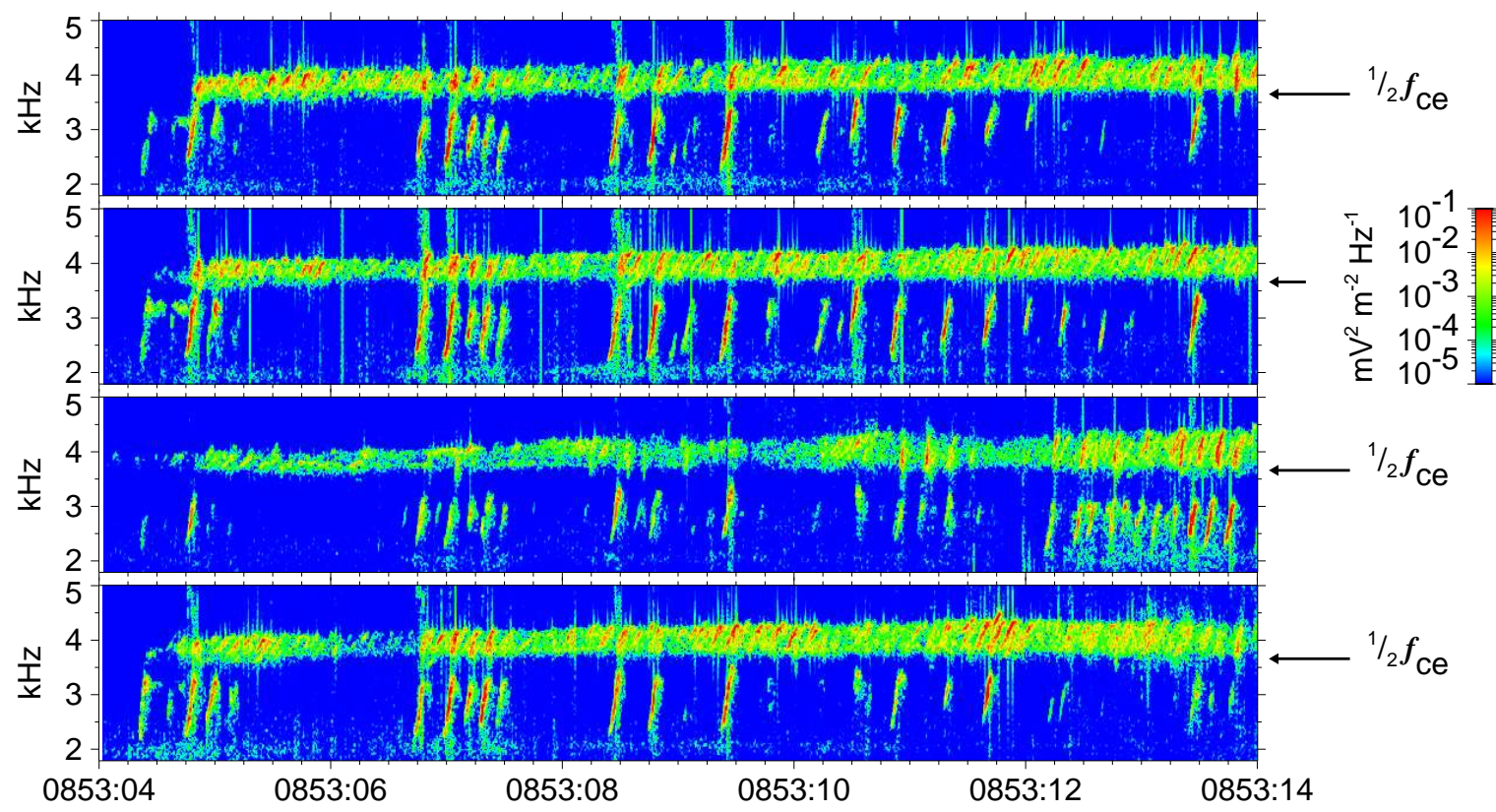

Fig. 3. The same as in Fig. 2 but for the time interval between 08:53:04 and 08:53:14 UT (interval I from Fig. 1).

(a)

$\frac{\bar{D}}{\frac{1}{0}}$

$\frac{\bar{v}}{\frac{0}{0}}$

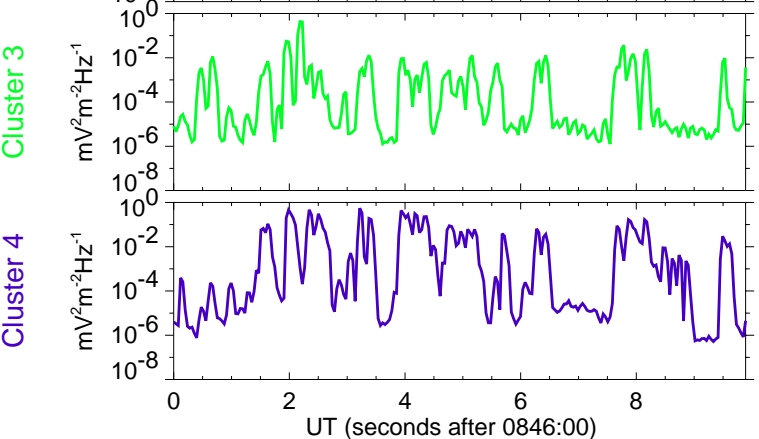

(b)

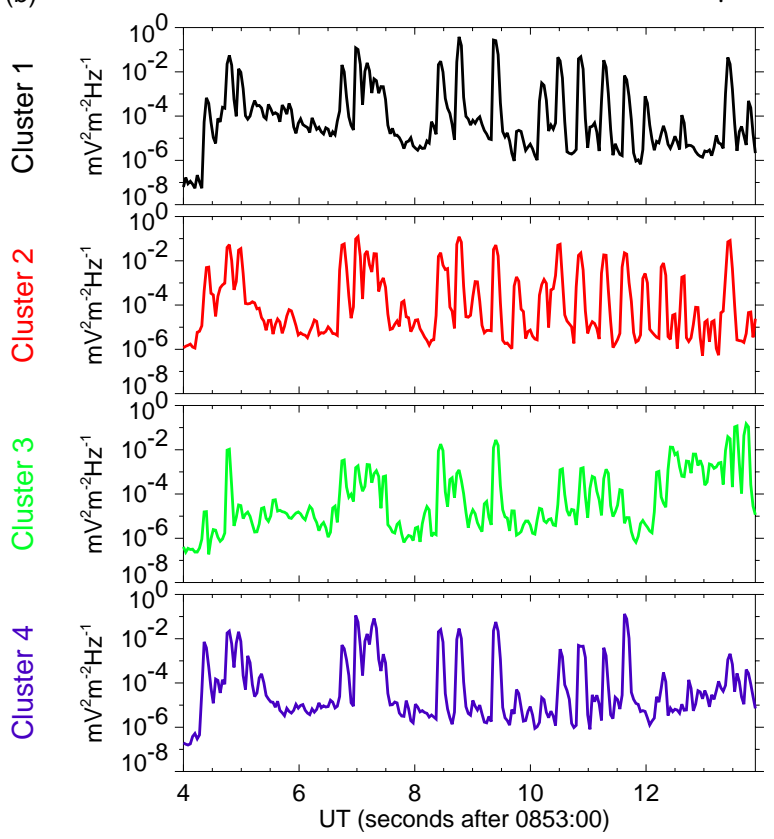

Fig. 4. Time series of power-spectral densities averaged in the frequency interval between $0.48 f_{c e}-1 \mathrm{kHz}$ and $0.48 f_{c e}$ (see text), for the time intervals in Fig. 2 (panel a), and Fig. 3 (panel b). The results for the four Cluster spacecraft are color coded.

and 2. On another example case, these differences have been quantified by means of the correlation analysis (Santolík and Gurnett, 2003). In the present paper, we use the same procedure, calculating averaged power-spectral densities in the $1-\mathrm{kHz}$ frequency band below the local $0.48 f_{c e}$. With the time resolution of $0.04 \mathrm{~s}$, this averaging gives a time series of 250 data points for each spacecraft in a 10-s time interval. The resulting time series are shown in Fig. 4 for the intervals from Figs. 2 and 3. The discrete chorus elements appear as peaks of enhanced power-spectral density, reaching the level of $1 \mathrm{mV}^{2} \mathrm{~m}^{-2} \mathrm{~Hz}^{-1}$. The background, mainly determined by the chorus-related hiss emissions, exceeds the level of the instrumental noise by several orders of magnitude, with powerspectral densities fluctuating below $10^{-4} \mathrm{mV}^{2} \mathrm{~m}^{-2} \mathrm{~Hz}^{-1}$. 
(a)

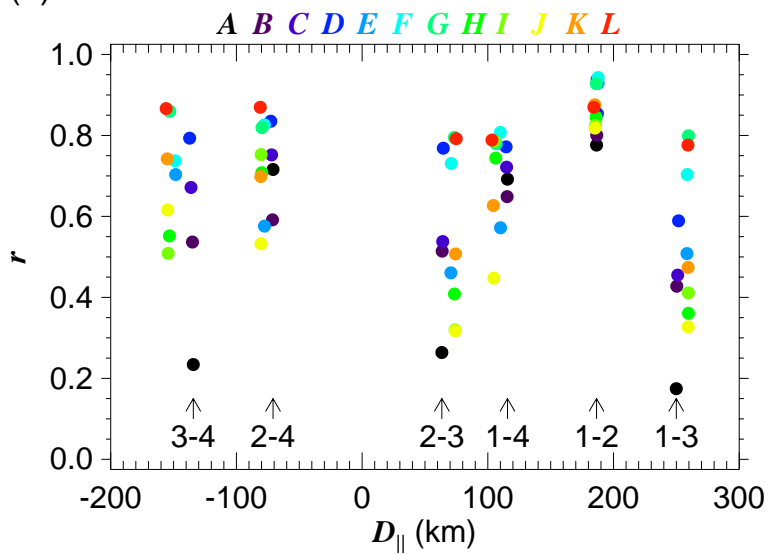

(b)

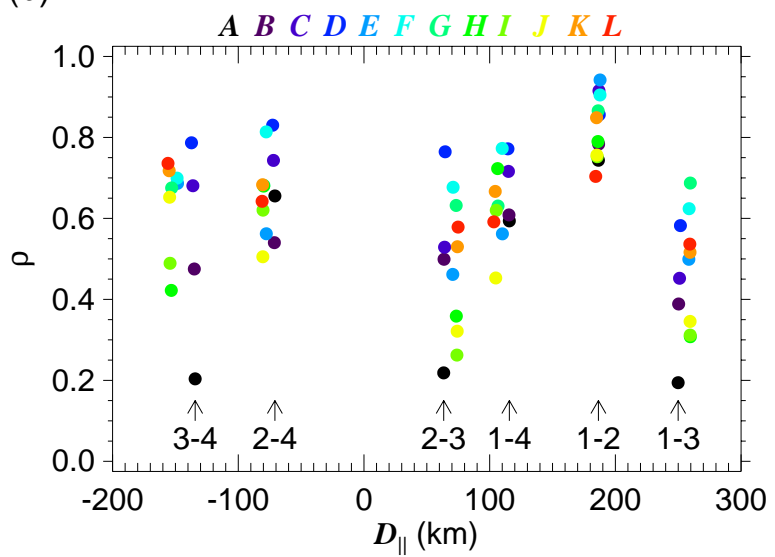

Fig. 5. The Pearson's coefficient $r$ of linear correlation (a) and the Spearman's rank correlation coefficient $\rho$ (b) as a function of the parallel separation. Colors indicate the twelve time intervals from Fig. 1, as listed on the top of each figure. The groups of points belonging to different spacecraft pairs are marked on the bottom.

(a)

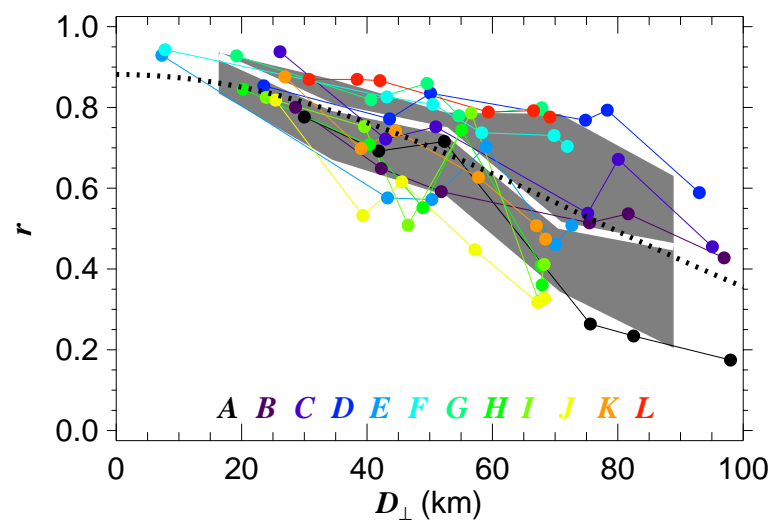

(b)

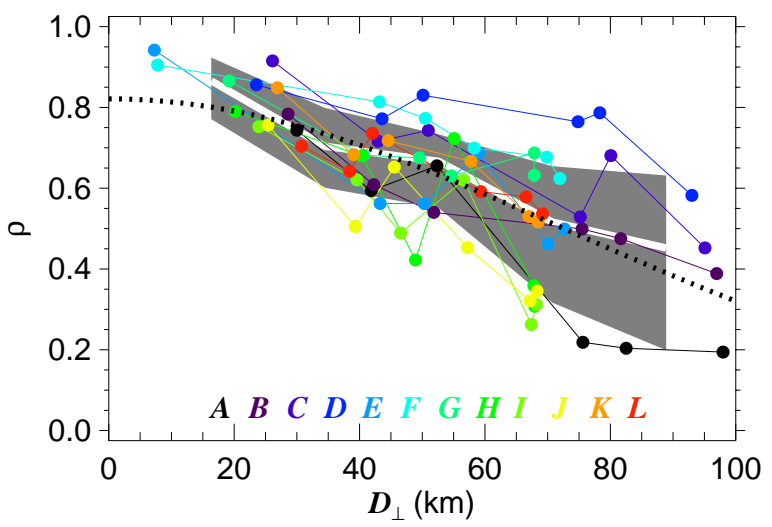

Fig. 6. Correlation coefficients $r$ (a) and $\rho$ (b) as a function of the perpendicular separation. Colors indicate the different time intervals. A solid line always connects the six values from the same interval. Shaded areas are drawn between the 0.16-quantile and 0.84-quantile, white line in the middle showing the median (0.5-quantile) values. Fit of a Gaussian function to all the points is plotted as a dotted line.

\section{Variation of the correlation coefficients of the fre- quency averaged power-spectral density}

The time series shown in Fig. 4 are used in the correlation analysis. We first calculate common logarithms $\mathcal{L}_{i \xi}$ of the frequency-averaged power-spectral densities for each spacecraft $i=1 \ldots 4$, where $\xi=1 \ldots N$ is the sample index in the time series, in our case $N=250$. We then calculate the Pearson's coefficient $r$ of linear correlation, and the nonparametric Spearman's rank correlation coefficient $\rho$ (e.g. Press et al., 1992). The Pearson's coefficient $r$ is, for a spacecraft pair $(i, j)$, given by the standard formula,

$r_{i j}=\frac{\sum_{\xi}\left(\mathcal{L}_{i \xi}-\overline{\mathcal{L}}_{i}\right)\left(\mathcal{L}_{j \xi}-\overline{\mathcal{L}}_{j}\right)}{\sqrt{\sum_{\xi}\left(\mathcal{L}_{i \xi}-\overline{\mathcal{L}}_{i}\right)^{2}} \cdot \sqrt{\sum_{\xi}\left(\mathcal{L}_{j \xi}-\overline{\mathcal{L}}_{j}\right)^{2}}}$,

where $\overline{\mathcal{L}}_{i}$ is the mean value of the $i$ th series, $\overline{\mathcal{L}}_{i}=\sum_{\xi} \mathcal{L}_{i \xi} / N$. The Spearman's rank correlation coefficient $\rho$ is calculated from the modified time series $\ell_{i \xi}$, constructed as magnitudebased ranks among the original series $\mathcal{L}_{i \xi}$,

$\rho_{i j}=\frac{\sum_{\xi}\left(\ell_{i \xi}-\bar{\ell}_{i}\right)\left(\ell_{j \xi}-\bar{\ell}_{j}\right)}{\sqrt{\sum_{\xi}\left(\ell_{i \xi}-\bar{\ell}_{i}\right)^{2}} \cdot \sqrt{\sum_{\xi}\left(\ell_{j \xi}-\bar{\ell}_{j}\right)^{2}}}$,

where $\bar{\ell}_{i}$ is the mean value of the series $\ell_{i \xi}$.

With the four spacecraft, we have six different pairs and therefore six values $r_{i j}$ of the Pearson's correlation coefficient $r$, and six values $\rho_{i j}$ of the Spearman's rank correlation coefficient $\rho$, i.e. 72 total values of each coefficient from the 12 selected intervals A-L (see Fig. 1). These intervals contain similar emissions, as shown in Figs. 2 and 3, and their selection is the same as in the previous analysis (Fig. 3 of Santolík and Gurnett, 2003), excluding the example interval from Fig. 1 of Santolík and Gurnett (2003). This selection was dictated by the requirement that separate wave packets of chorus have to appear as distinct peaks in the time series 
$\mathcal{L}_{i \xi}$. In all the other time intervals in the source region, either steady hiss-like emission is recorded, or the chorus elements can be recognized on the spectrograms but they follow each other so closely that it is impossible to distinguish them individually in the frequency-averaged time series. A different analysis method would be necessary for these intervals and, to allow comparison of our results with the previous analysis (Santolík and Gurnett, 2003), we have chosen the same analysis method, and therefore the same set of intervals.

The 72 resulting values of both correlation coefficients (Eqs. 1, 2) are plotted in Fig. 5 as a function of the parallel separation $D_{\|}$of the two spacecraft $(i, j)$. This separation is calculated as the component of their separation vector $\boldsymbol{D}$ in the direction parallel to $\boldsymbol{B}_{\mathbf{0}}, D_{\|}=\left(\boldsymbol{D} \cdot \boldsymbol{B}_{\mathbf{0}}\right) /\left|\boldsymbol{B}_{\mathbf{0}}\right|$. Different colors in Fig. 5 correspond to the 12 selected time intervals A-L. All the obtained values of $r$ and $\rho$ are significantly deviated from zero. The standard deviation of $r$ for uncorrelated data would be, roughly estimated, $\approx 0.06$, which is approximately 3 times lower than the lowest $r$ values obtained. The probability of obtaining the lowest observed $\rho$ values, assuming the hypothesis of uncorrelated data, is below $0.2 \%$. This means that the data are significantly correlated in all cases. We can see that the points are grouped according to the pairs of spacecraft, as identified on the bottom, i.e. for a given spacecraft pair, $D_{\|}$is nearly constant in the twelve time intervals. However, we cannot distinguish any trend of either correlation coefficient as a function of $D_{\|}$. Our interpretation is that the correlation does not depend on the parallel separation in our range of $D_{\|}$.

Figure 6 shows the results as a function of the perpendicular separation $D_{\perp}$, calculated as the modulus of the projection of the separation vector $\boldsymbol{D}$ to the plane perpendicular to $\boldsymbol{B}_{\mathbf{0}}, D_{\perp}=\left|\boldsymbol{D} \times \boldsymbol{B}_{\mathbf{0}}\right| /\left|\boldsymbol{B}_{\mathbf{0}}\right|$. The results are again plotted by different colors for the different intervals, the points from individual intervals being additionally connected by solid lines. In this case, a global trend of decreasing correlation coefficients with increasing $D_{\perp}$ can be seen. To investigate this global behavior, we have divided the total range of $D_{\perp}$ into 5 subranges, $0-20 \mathrm{~km}, 20-40 \mathrm{~km}, 40-60 \mathrm{~km}, 60-80 \mathrm{~km}$, and $80-100 \mathrm{~km}$. From all the values obtained in each subrange we have calculated the median value, the 0.16-quantile (corresponding to the mean value minus one standard deviation for the normal distribution), and the opposite 0.84-quantile. These robust statistical parameters clearly demonstrate the trend of obtained values of the correlation coefficients, as shown by the shaded areas in Fig. 6. We therefore choose model functions,

$r=A_{r} \exp \left(-\frac{D_{\perp}^{2}}{\Delta_{r}^{2}}\right), \quad \rho=A_{\rho} \exp \left(-\frac{D_{\perp}^{2}}{\Delta_{\rho}^{2}}\right)$,

allowing us to describe the decreasing trend quantitatively. Results of nonlinear least-squares fits of these two functions to all the data points are shown by dotted lines in Figs. 6a and $6 \mathrm{~b}$, respectively. The resulting optimized parameters are $A_{r}=0.88 \pm 0.01, \Delta_{r}=(105 \pm 3) \mathrm{km}$ for the Pearson's correlation coefficient, and $A_{\rho}=0.82 \pm 0.02, \Delta_{\rho}=(103 \pm 3) \mathrm{km}$ for the Spearman's rank correlation coefficient. In both cases, the error estimates are based on an assumption that all the obtained results have normally distributed random errors with a standard deviation of 0.06 . As it was already discussed in Sect. 3 of Santolík and Gurnett (2003), the results with $A_{r}<1, A_{\rho}<1$ mean that zero $D_{\perp}$ does not imply absolutely correlated data. Among the reasons could be the modulation by the spacecraft spin motion, clipping of the waveforms by the finite dynamics of the measurement, slight fluctuations of the $\boldsymbol{B}_{\mathbf{0}}$ direction, or, finally, small variations of the received power with $D_{\|}$. Note also that the model in Eq. 3 was chosen rather arbitrarily, and another function could be used to fit the data with a similar success, for example, an exponential function $\exp \left(-D_{\perp} / \delta\right)$.

Although we can see in Fig. 6 that the obtained values systematically decrease with increasing $D_{\perp}$, the results are scattered across rather large intervals of $r$ and/or $\rho$, especially for larger $D_{\perp}$. This could have two possible reasons: (1) variations of the characteristic correlation scale in the different time intervals, (2) variations of the correlation coefficient owing to the statistical nature of the source. In the remainder of this section we will examine the possibility (1), while the explanation (2) will be investigated in Sect. 4. Comparing now the results from the different time intervals with the global model (dotted line in Fig. 6) or median values (thick white line), we can see that the six points in every single interval sometimes are both above and below the model curve, for example, the results from the interval E. However, there are also intervals which consistently give results below or above the model, for example, the intervals D (above) and $\mathbf{J}$ (below). In some cases, we can find differences in this behavior comparing the two correlation coefficients, see, for example, the results from interval $\mathrm{L}$.

To quantify the differences between the results from the separate intervals, we have calculated the optimized parameters of the Gaussian model (Eq. 3) for each interval separately. We have used the same procedure as we used to obtain the global parameters shown in Fig. 6, but every time for just the six points from every single interval. The results are shown in Fig. 7. The relatively large error bars correspond to the low number of input points, but we still can see that there are nonnegligible differences between the results from the different time intervals. The characteristic scales of both correlation coefficients can thus be found in an extended interval $\approx 60-200 \mathrm{~km}$. We do not observe any clear signs of ordering of these results with respect to the parallel distance from the central position inside the source.

\section{Interpretation using a 2-D model of the source region}

In an attempt to investigate the statistical nature of our results, we have developed a simple computational model of the chorus source region. We realize that the actual configuration is necessarily much more complex than what we assume below, but we'll demonstrate that even such a simple 
(a)

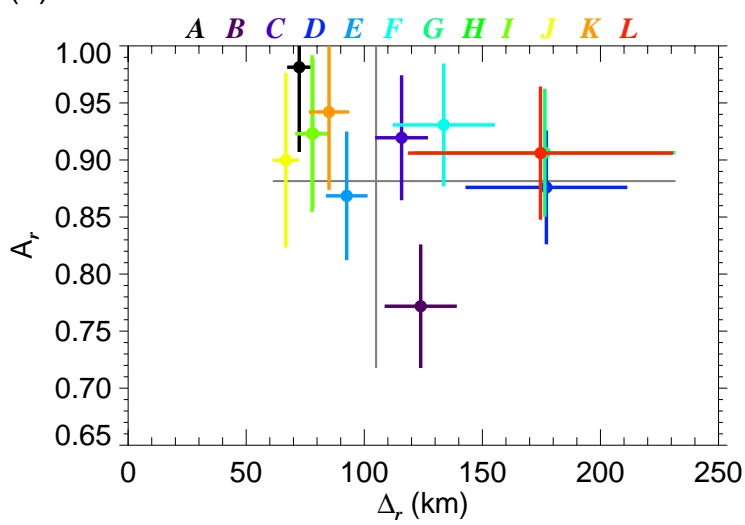

(b)

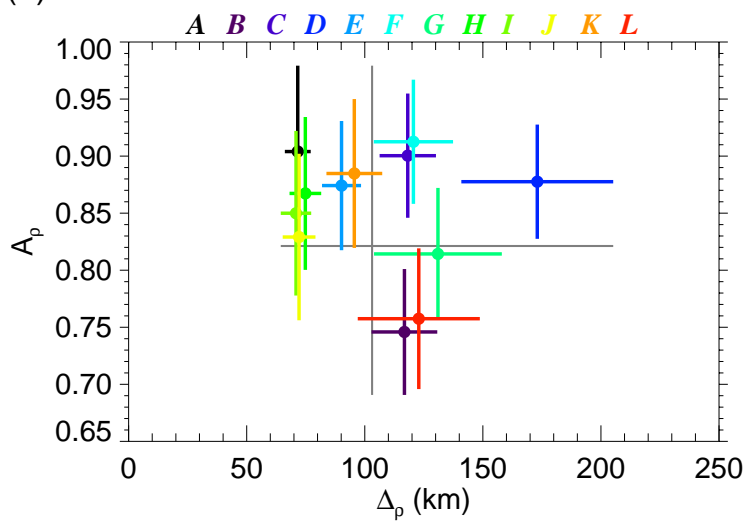

Fig. 7. Parameters of the Gaussian model (Eq. 3) estimated from each time interval separately. Results for both the coefficient $r$ of linear correlation (a) and the rank correlation coefficient $\rho$ (b) are given. Error bars correspond to estimates of the standard deviations of the results. Colors coded are the twelve different time intervals as listed on the top of each figure. Positions of the thin gray lines indicate the global parameters obtained from all the intervals; their length is spanned over the total range of obtained results.

(a)

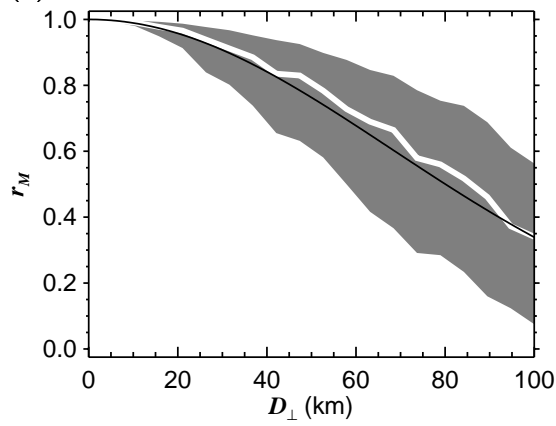

(b)

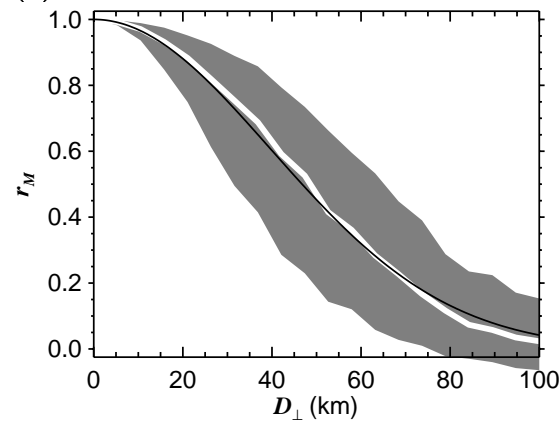

(c)

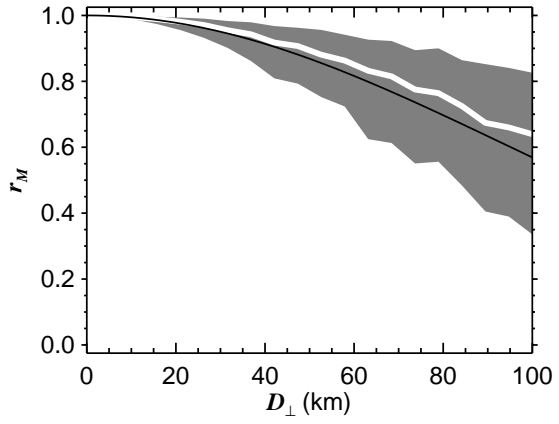

Fig. 8. Pearson's correlation coefficient $r_{M}$ of simulated model data as a function of $D_{\perp}$, obtained using the same analysis method as in Fig. 6a. The same quantiles as in Fig. 6 are shown by the shaded areas. Fits of Gaussian functions (Eq. 3) are shown by dark solid lines. Simulation (see text) with (a) $d_{i}=35 \mathrm{~km}$, (b) $d_{i}=20 \mathrm{~km}$, (c) $d_{i}=50 \mathrm{~km}$.

model predicts statistical properties of the correlation coefficient which are surprisingly close to the observations.

We suppose that the individual wave packets of chorus are generated in localized areas whose central positions $\boldsymbol{X}_{\boldsymbol{i}}$ are randomly distributed in a 2-D plane perpendicular to $\boldsymbol{B}_{\mathbf{0}}$. Each location has a 2-D distribution of radiated power decreasing as a Gaussian function with the distance of the point of observation $\boldsymbol{x}$ from the central position,

$P(x)=P_{i} \exp \left(-\frac{\left|\boldsymbol{x}-\boldsymbol{X}_{\boldsymbol{i}}\right|_{\perp}^{2}}{d_{i}^{2}}\right)$

where $P$ is the wave power at position $\boldsymbol{x}, d_{i}$ is the half-width of the active area, and $P_{i}$ characterizes its strength. In our model we assume that all the individual active areas have the same $P_{i}$ and $d_{i}$. Each of the active areas starts its radiation at a random time $t_{0}$ and the wave power is detected by a pair of virtual spacecraft with a predefined perpendicular separation $D_{\perp}$. In this simple 2-D model we suppose that the two spacecraft are close to the source and we do not take into account any propagation effects. This assumption is equivalent to the straight line propagation perpendicular to the source plane (i.e., along $\boldsymbol{B}_{\mathbf{0}}$ ). This is most probably close to reality, since the group velocity of the whistler mode does not deviate very much from $\boldsymbol{B}_{\mathbf{0}}$, even if the emission is emitted in a wide interval of wave vector directions. This might well be the case, taking into account the finite transverse dimension of the sources. The finite interval of angles between emitted rays and $\boldsymbol{B}_{\mathbf{0}}$ might then slightly increase the observed correlation coefficients with respect to this simple model.

To account for the quasi-periodic occurrence of the wave packets, we suppose that each active area radiates a series of wave packets, where the neighbor wave packets are separated by a randomly chosen delay $\tau$ between 0.2 and $0.6 \mathrm{~s}$. The number $n$ of wave packets in a series is chosen randomly between 1 and 8 , the duration of every wave packet being set to a fixed value of $0.08 \mathrm{~s}$. These numerical values roughly correspond to the parameters of chorus during the selected intervals which have been used for analysis in Fig. 6a. The background noise is supposed to be at the level of $10^{-4} P_{i}$, leading to a "visibility circle" with a radius of $\sqrt{ } \ln \left(10^{4}\right) \cdot d_{i} \approx 3 d_{i}$ around the position of each virtual spacecraft. The central 
position $\boldsymbol{X}_{\boldsymbol{i}}$ must appear inside the visibility circle if the wave packets from an active area are to be detected above the noise level at a given spacecraft. The spatial and temporal density of such randomly generated active areas is adjusted to obtain, on average, one area acting at a time within the visibility circle. The model doesn't include any of the possible factors decreasing the correlation at low $D_{\perp}$ mentioned in Sect. 3 .

Our simulation procedure then consists in (1) defining positions of a pair of virtual spacecraft consistent with a perpendicular separation $D_{\perp}$, (2) generating $10 \mathrm{~s}$ of artificial data, i.e. for each spacecraft, 250 samples of wave power with the sampling period of $0.04 \mathrm{~s}$, and with statistical properties defined by the above described model, (3) calculating the Pearson's coefficient of linear correlation $r_{M}$ in the same way as we did with the experimental data using Eq. 1, (4) repeating items 2 and 3 for 500 independent realizations of data with the random parameters $\boldsymbol{X}_{\boldsymbol{i}}, t_{0}, \tau$, and $n$. Results are shown in Figs. 8a-c for three different fixed values of $d_{i}$. Twenty predefined values of $D_{\perp}$, from 0 to $100 \mathrm{~km}$, are used in each figure. For a given $D_{\perp}, 0.16,0.5$, and 0.84 quantiles are calculated from each set of 500 realizations of $r_{M}$, and shown in the same way as in Fig. 6a. Fits of a free parameter $\Delta_{r}$ from Eq. 3 to all $20 \times 500$ simulated data points in each figure show that the median value is always close to a Gaussian function of $D_{\perp}$. The obtained numerical values for $\Delta_{r}$ are found close to $2.7 d_{i}$, while $A_{r}=1$, according to the definition of this simplified model.

The obtained statistical properties of the simulated data indicate that the large spread of $r$, obtained from the observations (Fig. 6a), can be reproduced just by a random spatial and temporal distribution of localized generation areas, while assuming the same fixed half-width $d_{i}$ for all of them. This behavior can be intuitively described, realizing that the position of the simulated active areas is random with respect to the two virtual spacecraft. If an active area accidentally appears exactly in the middle between them, with its central position lying on the same line as the spacecraft, the simulated signals will be absolutely correlated. If, on the other hand, an active area appears much closer to one of the virtual spacecraft, the correlation will be low. Those differences then cause the large statistical spread of the obtained results. Comparison with Fig. 6a shows that the model with $d_{i}=35 \mathrm{~km}$ (Fig. 8a) is the closest one to the observed data. This is consistent with the above mentioned numerical results on $\Delta_{r}$, which is close to the observed values of $\approx 100 \mathrm{~km}$ for $d_{i}=35 \mathrm{~km}$.

\section{Conclusions}

This work is a continuation of a previous research (Santolík and Gurnett, 2003) on the correlation of the frequency averaged power-spectral density of chorus in its source region. It was shown that the correlation coefficients decrease with increasing separation of spacecraft in the plane perpendicular to the static magnetic field. In this plane, the characteristic scale of the changes of the correlation coefficients was found to be close to $100 \mathrm{~km}$, The characteristic scale parallel to the static magnetic field is at least several times higher. However, the values of the correlation coefficient obtained at similar perpendicular separations were found to be significantly scattered when combining the result from different time intervals within the source region.

In the present paper we have investigated possible explanations for this large spread of obtained values of both the Pearson's coefficient of linear correlation, and the Spearman's rank correlation coefficient. Our conclusion is that two different effects may act simultaneously.

(1) The perpendicular scale of the correlation coefficients varies approximately between 60 and $200 \mathrm{~km}$ when comparing different data intervals inside the same source region. No systematic variations of the perpendicular scale with respect to the parallel position within the source region have been found.

(2) The individual chorus wave packets may be generated at random places within a broader source region. When detected by a pair of spacecraft at a given perpendicular separation, this randomness causes a random spread of the obtained values of the correlation coefficients. A simple 2-D model of statistical properties of the source region approximately reproduces the observations if we assume a perpendicular half-width of $35 \mathrm{~km}$ for the power carried by individual wave packets. This half-width is comparable to the wavelength of the radiated wave.

The individual wave packets are thus radiated from regions with very small characteristic perpendicular dimensions with respect to the field lines in the equatorial region. This most probably implies that the distribution of the initial wave normal angles is rather wide in the source region. On the other hand, the parallel scales of these source regions can be tens to hundreds times larger, forming long and narrow spaghettilike objects. The question is whether those very low perpendicular scales are the general property of the chorus source mechanism, or just a special case of the presented observations under the disturbed magnetospheric conditions. A systematic study of a set of similar multipoint observations is under way.

Acknowledgements. We thank R. Huff, J. Dowell, J. Seeberger and other colleagues from the University of Iowa for the calibration and preprocessing of the WBD measurements. We acknowledge discussions of the STAFF data with Nicole Cornilleau-Wehrlin of CETP Velizy (PI of the STAFF instrument), M. Parrot of LPCE Orleans, C. Harvey of CESR Toulouse, M. Maksimovic of the Meudon Observatory, and other colleagues from the STAFF team. We thank P. Canu and P. Décréau (PI of Whisper) for analyzing the Whisper data. We acknowledge discussions with A.G. Demekhov and V.Y. Trakhtengerts of IAP Nizhny Novgorod, and the access to the spin-resolution data of the FGM magnetic field experiment (PI A. Balogh) used for reference. This research was supported by the NASA Goddard Space Flight Center under Grant No. NAG5-9974 and by the NSF award No. 0307319 / ME 650. O. Santolík acknowledges additional support from grants MSM 113200004 and GACR 202/03/0832.

Topical Editor T. Pulkkinen thanks N. Cornilleau-Wehrlin and another referee for their help in evaluating this paper. 


\section{References}

Balogh, A., C. M. Carr, M. H. Acuña, et al., The Cluster Magnetic Field Investigation: overview of in-flight performance and initial results Ann. Geophys., 19, 1207-1217, 2001.

Burtis, W. J. and R. A., Helliwell: Banded chorus - a new type of VLF radiation observed in the magnetosphere by OGO 1 and OGO 3, J. Geophys. Res., 74, 3002-3010, 1969.

Burton, R. K. and Holzer, R. E.: The origin and propagation of chorus in the outer magnetosphere, J. Geophys. Res., 79, 10141023, 1974.

Canu, P., Décréau, P. M. E., Trotignon, J. G. et al.: Identification of natural plasma emissions observed close to the plasmapause by the Cluster-Whisper relaxation sounder Ann. Geophys., 19, 1697-1709, 2001.

Cornilleau-Wehrlin, N., Chanteur G., Perraut S. et al.: First results obtained by the Cluster STAFF experiment, Ann. Geophys. 21, 437-456, 2003.

Décréau, P. M. E., Fergeau, P., Krasnoselskikh, V. et al.: Early results from the Whisper instrument on Cluster: An overview Ann. Geophys., 19, 1241-1258, 2001.

Goldstein, B. E. and Tsurutani, B. T.: Wave normal directions of chorus near the equatorial source region, J. Geophys. Res., 89, 2789-2810, 1984.

Gurnett, D. A., Huff R. L., and Kirchner, D. L.: The wide-band plasma wave investigation, Space Sci. Rev., 79, 195-208, 1997.

Gurnett, D. A., Huff, R. L., Pickett, J. S. et al.: First results from the Cluster wideband plasma wave investigation, Ann. Geophys., 19, 1259-1272, 2001.

Hattori, K., Hayakawa, M., Lagoutte, D., Parrot, M., and Lefeuvre, F.: Further evidence of triggering chorus emissions from wavelets in the hiss band, Planet. and Space Sci., 39, 1465-1472, 1991.

Hayakawa, M., Yamanaka, Y., Parrot, M., and Lefeuvre, F.: The wave normals of magnetospheric chorus emissions observed on board GEOS 2, J. Geophys. Res., 89, 2811-2821, 1984.

Helliwell, R. A.: A theory of discrete emissions from the magnetosphere, J. Geophys. Res., 72, 4773-4790, 1967.

Horne, R. B., Glauert, S. A., and Thorne, R. M.: Resonant diffusion of radiation belt electrons by whistler-mode chorus, Geophys. Res. Lett., 30(9), 1493, doi:10.1029/2003GL016963, 2003.

Horne, R. B. and R. M. Thorne: Relativistic electron acceleration and precipitation during resonant interactions with whistler-mode chorus, Geophys. Res. Lett., 30(10), 1527, doi:10.1029/2003GL016973, 2003.
Kennel, C. F. and Petschek, H. E.: Limit on stable trapped particle fluxes, J. Geophys. Res., 71, 1-28, 1966.

Koons, H. C.: The role of hiss in magnetospheric chorus emissions, J. Geophys. Res., 86,6745-6754, 1981.

Lauben, D. S., Inan, U. S., Bell, T. F., Kirchner, D. L., Hospodarsky, G. B., and Pickett, J. S.: VLF chorus emissions observed by polar during the 10 January 1997 magnetic cloud, Geophys. Res. Lett., 25, 2995-2998, 1998.

LeDocq, M. J., Gurnett, D. A., and Hospodarsky, G. B.: Chorus source locations from VLF poynting flux measurements with the polar spacecraft, Geophys. Res. Lett., 25, 4063-4066, 1998.

Meredith, N. P.,Cain, M., Horne, R. B., Thorne, R. M., Summers, D., and Anderson, R. R.: Evidence for chorus-driven electron acceleration to relativistic energies from a survey of geomagnetically disturbed periods, J. Geophys. Res., 108(A6), 1248, doi:10.1029/2002JA009764, 2003.

Nunn, D.,Omura, Y.,Matsumoto, H., Nagano, I., and Yagitani, S.: The numerical simulation of VLF chorus and discrete emissions observed on the Geotail satellite using a Vlasov code, J. Geophys. Res., 102(A12), 27 083-27 097, 1997.

Omura, Y., Nunn, D., Matsumoto, H., and Rycroft, M. J.: A review of observational, theoretical and numerical studies of VLF triggered emissions, J. Atmos. and Terr. Phys., 53, 351-368, 1991.

Parrot, M., Santolík, O., Cornilleau-Wehrlin, N. Maksimovic, M., Harvey, C.: Source location of chorus emissions observed by CLUSTER, Ann. Geophys., 21(2), 473-480, 2003.

Press, W. H., Flannery, B. P., Teukolsky, S. A., and Vetterling, W. T.: Numerical Recipes, Cambridge Univ. Press, New York, 1992.

Santolík, O. and Gurnett, D. A.: Transverse dimensions of chorus in the source region, Geophys. Res. Lett., 30(2), 1031, doi:10.1029/2002GL016178, 2003.

Santolík, D., Gurnett, A.,Pickett, J. S., Parrot, M., and CornilleauWehrlin, N.: Spatio-temporal structure of storm-time chorus, J. Geophys. Res., 108(A7), 1278, doi:10.1029/2002JA009791, 2003.

Stix, T. H.: Waves in Plasmas, Am. Inst. of Phys., New York, 1992.

Sazhin, S. S. and Hayakawa, M.: Magnetospheric chorus emissions: A review, Planet. Space Sci., 40, 681-697, 1992.

Trakhtengerts, V. Y.: A generation mechanism for chorus emission, Ann. Geophys., 17, 95-100, 1999.

Tsurutani, B. T. and Smith, E. J.: Postmidnight chorus: a substorm phenomenon, J. Geophys. Res., 79, 118-127, 1974. 